Discrete Comput Geom 34:507-521 (2005)

DOI: $10.1007 / \mathrm{s} 00454-005-1193-\mathrm{z}$

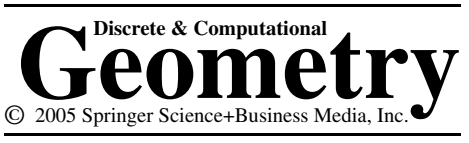

\title{
Families of Tight Inequalities for Polytopes
}

\author{
Catherine Stenson \\ Department of Mathematics, Juniata College, \\ Huntingdon, PA 16652, USA \\ stenson@juniata.edu
}

\begin{abstract}
We use the Billera-Liu algebra to show how the flag $f$-vectors of several special classes of polytopes fit into the closed convex hull of the flag $f$-vectors of all polytopes. In particular, we describe inequalities that define the faces of the closed convex hull of the flag $f$-vectors of all $d$-polytopes that are spanned by the flag $f$-vectors of simplicial, simple, $k$-simplicial, and $k$-simple $d$-polytopes. We also describe inequalities that define the face of the closed convex hull of the flag $f$-vectors of all $d$-zonotopes spanned by the flag $f$-vectors of cubical $d$-zonotopes, and give an upper bound on the dimension of the span of the flag $f$-vectors of $k$-cubical zonotopes. Finally, we strengthen some previously known inequalities for flag $f$-vectors of zonotopes.
\end{abstract}

\section{Introduction}

The flag $f$-vector of a polytope contains much of the combinatorial information about that polytope. It is a generalization of the classic idea of counting vertices, edges, and faces. Steinitz [24] characterized these counts (the $f$-vector) for three-dimensional polytopes. This translates to a characterization of flag $f$-vectors of 3 -polytopes. Dimensions $d \geq 4$ are still open.

Considerable progress has been made on several aspects of this problem. Bayer and Billera [3] introduced the generalized Dehn-Sommerville equations and showed that they are the only linear equations satisfied by the flag $f$-vectors of all polytopes. Their results show that the convex hull of the flag $f$-vectors of all $d$-polytopes has dimension $a_{d}-1$, where $a_{d}$ is the $d$ th Fibonacci number $\left(a_{0}=1, a_{1}=1\right)$.

Fine (unpublished) and Bayer and Klapper [4] showed that the flag $f$-vector could be written in terms of the cd-index, and Stanley [23], Billera and Ehrenborg [6], and Ehrenborg [16] used the cd-index to derive many linear inequalities satisfied by the flag $f$-vectors of all polytopes. Stanley [22] also examined the toric $g$-vector, whose entries are linear combinations of entries of the flag $f$-vector, and showed that it is non-negative for all rational polytopes. Karu [19] showed that it is non-negative for all polytopes. 
Another approach to the flag $f$-vector problem is to consider special classes of polytopes. The flag $f$-vectors of simplicial (dually, simple) polytopes were characterized by Billera and Lee [11], [12] and Stanley [21] in their proof of a conjecture of McMullen. Adin [1] introduced the cubical $h$-vector, which provides insight into the flag $f$-vectors of cubical polytopes. Billera et al. [8] showed that flag $f$-vectors of a class of polytopes known as zonotopes do not satisfy any linear equations beyond the generalized DehnSommerville equations. Varchenko [25] and Liu [20] found a class of linear inequalities satisfied by flag $f$-vectors of zonotopes but not all polytopes.

In this paper we show how the flag $f$-vectors of these special classes of polytopes fit into the convex hull of the flag $f$-vectors of all polytopes. In Section 2 we examine the relationship between equalities satisfied by simplicial $d$-polytopes (dually, simple $d$-polytopes) and inequalities satisfied by lower-dimensional polytopes. We give inequalities that define the simplicial face of the convex hull of flag $f$-vectors. In Section 3 we extend this work to $k$-simplicial and $k$-simple polytopes, and in Section 4 we use the same approach to examine the relationship between equalities satisfied by cubical and $k$-cubical zonotopes and inequalities satisfied by lower-dimensional zonotopes. Finally, in Section 5 we improve the Varchenko-Liu inequalities.

\section{Preliminaries}

A polytope $P$ is the convex hull of a finite set of points in Euclidean space. It is $d$ dimensional, or a $d$-polytope, if its affine span is $\mathbf{R}^{d}$. Its boundary is a collection of lower-dimensional polytopes known as the faces of $P$. Let $f_{i}(P)$ be the number of $i$ faces of $P$. We call $\left(f_{0}, f_{1}, \ldots, f_{d-1}\right)$ the $f$-vector of $P$. More generally, let $f_{i_{1} i_{2} \ldots i_{k}}$ be the number of chains of faces $F_{i_{1}} \subseteq F_{i_{2}} \subseteq \cdots \subseteq F_{i_{k}}$ in $P$ with $\operatorname{dim}\left(F_{i_{j}}\right)=i_{j}$. These $f_{S}$, where $S$ ranges over all subsets of $\{0,1, \ldots, d-1\}$, are the entries of the flag f-vector of $P$. We will often write $f_{S}^{d}$ when we wish to emphasize that $P$ is $d$-dimensional.

The first dimension in which there is more than one combinatorial type of polytope is $d=2$. All 2-polytopes are polygons. Their flag $f$-vectors are entirely determined by $f_{0}$, the number of vertices, and clearly all polygons satisfy $f_{0} \geq 3$.

Dimension 3 is more interesting. In this case the flag $f$-vector is determined by the ordinary $f$-vector.

Theorem 1 [24]. An integer vector $\left(f_{0}, f_{1}, f_{2}\right)$ is the $f$-vector of a 3-polytope if and only if it satisfies the Euler relation $f_{2}-f_{1}+f_{0}=2$ and

(i) $f_{0} \leq 2 f_{2}-4$,
(ii) $f_{2} \leq 2 f_{0}-4$.

How can we understand this result in terms of special families of polytopes? First, recall that a $d$-polytope is simplicial if all its facets, or $(d-1)$-faces, are simplices and cubical if all its facets are combinatorially equivalent to cubes. Dual to simplicial $d$-polytopes are simple $d$-polytopes, in which each vertex is incident with precisely $d$ facets. Finally, a zonotope is the Minkowski sum of a finite set of line segments. Note all faces of a zonotope are also zonotopes. 


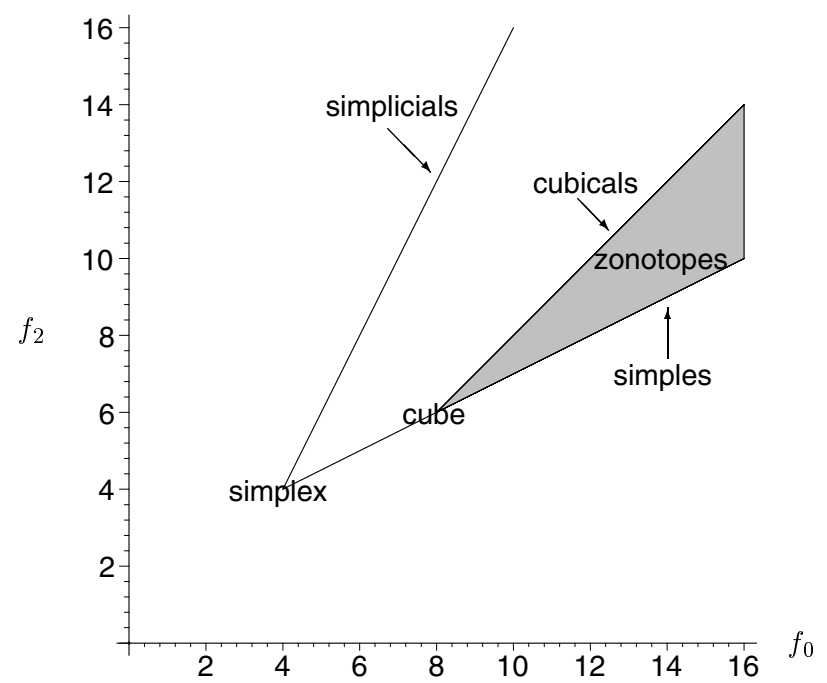

Fig. 1. The cone bounded by the simple and simplicial lines is the convex hull of the $f$-vectors of 3-polytopes. The shaded cone is the convex hull of the $f$-vectors of 3-zonotopes.

Each of the extreme rays of Steinitz's cone corresponds to a special family of polytopes: $f_{0}(P)=2 f_{2}(P)-4$ if and only if $P$ is simple, and $f_{2}(P)=2 f_{0}(P)-4$ if and only if $P$ is simplicial. The 3-zonotopes satisfy the stronger inequality $f_{2} \leq f_{0}-2$, and the cubical 3-polytopes (including the cubical 3-zonotopes) sit on the ray $f_{2}=f_{0}-2$. (See Fig. 1.) Note that the vertex of the polytope cone is the flag $f$-vector of the 3 -simplex and the vertex of the zonotope cone is the flag $f$-vector of the 3 -cube.

We can understand this picture in terms of the convolution operation defined by Kalai [18]. The convolution of $f_{S}^{m}$ and $f_{T}^{n}$ is

$$
f_{S}^{m} * f_{T}^{n}=\sum_{\substack{F \subset Q \\ \operatorname{dim}(F)=m}} f_{S}^{m}(F) f_{T}^{n}(Q / F),
$$

where $Q / F$ is the quotient of $Q$ by the face $F$ (see [27] for a discussion of quotient polytopes). Note this is just $f_{S \cup\{m\} \cup(T+m+1)}^{m+n+1}$, where $T+m+1=\{i+m+1 \mid i \in T\}$. Now extend this to linear forms $M=\sum_{S} \alpha_{S} f_{S}^{m}$ and $N=\sum_{T} \beta_{T} f_{T}^{n}\left(\alpha_{S}, \beta_{T} \in \mathbf{R}\right)$ by

$$
M * N=\sum_{\substack{F \subset Q \\ \operatorname{dim}(F)=m}} \sum_{S, T} \alpha_{S} \beta_{T} f_{S}^{m}(F) f_{T}^{n}(Q / F)=\sum_{S, T} \alpha_{S} \beta_{T} f_{S}^{m} * f_{T}^{n}
$$

If $M \geq 0$ for all $m$-polytopes and $N \geq 0$ for all $n$-polytopes, then $M * N \geq 0$ for all $(m+n+1)$-polytopes.

While $f_{\emptyset}^{d}=1$ for all $d$-polytopes, it is useful to consider it as a linear form in its own right. By doing so, we can see that $\left(f_{0}^{2}-3 f_{\emptyset}^{2}\right) * f_{\emptyset}^{0}=f_{02}^{3}-3 f_{2}^{3} \geq 0$ for all 3-polytopes. Rewritten in terms of $f_{0}$ and $f_{2}$ this becomes the second of Steinitz's inequalities. Since $f_{\emptyset}^{0}=1$ for all 0-polytopes, we can see that $\left(\left(f_{0}^{2}-3 f_{\emptyset}^{2}\right) * f_{\emptyset}^{0}\right)(P)=0$ precisely when $\left(f_{0}^{2}-3 f_{\emptyset}^{2}\right)(F)=0$ for each facet $F$ of $P$. This holds if and only if each facet of $P$ has three vertices, that is, when $P$ is simplicial. 
Dually, we see that $f_{\emptyset}^{0} *\left(f_{0}^{2}-3 f_{\emptyset}^{2}\right) \geq 0$ for all 3-polytopes; rewritten in terms of $f_{0}$ and $f_{2}$ this becomes the first of Steinitz's inequalities. By the same reasoning as above, this inequality is an equality for a polytope $P$ precisely when $\left(f_{0}^{2}-3 f_{\emptyset}^{2}\right)(F)=0$ for each $F=P / v$ for $v$ a vertex in $P$. This holds if and only if each vertex of $P$ has a triangular vertex figure, that is, when $P$ is simple.

We can understand the smaller zonotope cone in the same way. The 2-zonotope with the fewest possible vertices is a parallelogram, so $f_{0}-4 \geq 0$ for all 2-zonotopes. Since every facet of a 3-zonotope $Z$ must be a 2-zonotope, we have $\left(\left(f_{0}^{2}-4 f_{\emptyset}^{2}\right) * f_{\emptyset}^{0}\right)(Z) \geq 0$, which is equivalent to $f_{2} \leq f_{0}-2$. Note that we cannot tighten the simple inequality because vertex figures in zonotopes are not necessarily zonotopes. The cube and the prism over a hexagon are examples of simple zonotopes.

So we obtain the inequality that defines the simplicial face of the convex hull of the flag $f$-vectors of 3-polytopes by "lifting" (that is, convolving with $f_{\emptyset}^{0}$ ) the inequality that is satisfied by all 2-polytopes with equality on the 2-simplex. Similarly, we obtain the inequality that defines the cubical face of the convex hull of the flag $f$-vectors of 3 -zonotopes by lifting the inequality that is satisfied by all 2-zonotopes with equality on the 2-cube.

We show that this idea generalizes to higher dimensions. To do this, we need an algebra defined by Billera and Liu [13]. Let $A_{\mathcal{E}}$ be the Q-algebra of linear forms on Eulerian posets. Define $y_{i+1}=f_{\emptyset}^{i}$. (The subscript indicates rank rather than dimension, which is a more natural choice for posets. Note that face posets of polytopes are Eulerian.)

Theorem 2 [13]. There is a graded isomorphism of $\mathbf{Q}$-algebras

$$
A_{\mathcal{E}} \cong \mathbf{Q}\left\langle y_{1}, y_{3}, y_{5}, \ldots, y_{2 k+1}, \ldots\right\rangle \text {. }
$$

So every linear form has a unique representation in terms of the $y_{2 k+1}$ 's. We use this uniqueness to establish the independence of the linear forms that will define the simplicial face of the convex hull of the flag $f$-vectors of polytopes. Following Billera and Liu's notation, we omit the $*$ in convolutions of the $y_{2 k+1}$ 's. We also note that Bergeron et al. [5] showed that the Billera-Liu algebra is dual to Stembridge's algebra of peak quasisymmetric functions. This is part of a larger body of recent work on combinatorial Hopf algebras; see, for example, [2], [10], and [15].

\section{Simplicial and Simple Polytopes}

We have seen that in dimension 3, the simplicial and simple faces of the flag $f$-vector cone are defined by liftings of the dimension 2 inequality $f_{0}^{2}-3 f_{\emptyset}^{2} \geq 0$.

We show that this idea generalizes. Inequalities for flag $f$-vectors of $d$-polytopes that are equalities for simplicial $d$-polytopes are liftings of inequalities for $(d-j)$-polytopes $(j>0, j$ odd) that are equalities for the $(d-j)$-simplex.

More formally, let $Y^{d-j}$ be a linear form written in terms of the $y_{2 k+1}$ 's that is non-negative for all $(d-j)$-polytopes and 0 for the $(d-j)$-simplex. For each odd $j$ in $\{1, \ldots, d\}$, pick a set $R_{j}$ of $a_{d-j}-1$ linearly independent such $Y^{d-j}$, where $a_{d-j}$ is the $(d-j)$ th Fibonacci number. We know that these $Y^{d-j}$ exist because 
Billera and Ehrenborg [6] showed that the flag $f$-vector of the simplex is a vertex of the convex hull of flag $f$-vectors of polytopes. Let $S_{j}=\left\{Y^{d-j} y_{j} \mid Y^{d-j} \in R_{j}\right\}$. Let $S^{d}=\cup_{1 \leq j \leq d, j \text { odd }} S_{j}$.

Theorem 3. $S^{d}$ is a linearly independent set of linear forms that are non-negative for all d-polytopes and vanish on simplicial d-polytopes. The intersection of the zero sets of these forms is precisely the span of the flag $f$-vectors of simplicial polytopes.

Proof. Each $Y^{d-j} y_{j}$ is a convolution of non-negative forms and hence non-negative for all $d$-polytopes. Each is also 0 for any $d$-polytope whose $(d-j)$-faces are all simplices; this includes the simplicial $d$-polytopes.

Now we show linear independence. Suppose we have

$$
\sum_{\substack{1 \leq j \leq d \\ j \text { odd }}} \sum_{i=1}^{a_{d-j}-1} \alpha_{i}^{j} Y_{i}^{d-j} y_{j}=0
$$

where $\alpha_{i}^{j} \in \mathbf{R}$. Because every form has a unique representation in terms of the $y_{2 k+1}$ 's, we must have

$$
\sum_{i=1}^{a_{d-j}-1} \alpha_{i}^{j} Y_{i}^{d-j} y_{j}=0
$$

for each $j$. However, that can only happen if $\alpha_{i}^{j}=0$ for all $i, j$ since each $R_{j}$ is linearly independent. So $S^{d}$ is linearly independent.

Now we show that $S^{d}$ has the desired number of elements. The affine span of the flag $f$-vectors of simplicial $d$-polytopes has dimension $\lfloor d / 2\rfloor$ [17, Section 9.2]. We know $\left|S^{d}\right|=\sum_{1 \leq j \leq d, j \text { odd }}\left(a_{d-j}-1\right)$. We can simplify this expression by writing $a_{d}$ in terms of smaller Fibonacci numbers. If $d$ is odd, then $a_{d}=a_{d-1}+a_{d-3}+\cdots+a_{2}+a_{0}$. If $d$ is even, then $a_{d}=a_{d-1}+a_{d-3}+\cdots+a_{1}+1$. So if $d$ is odd,

$$
\begin{aligned}
\sum_{\substack{1 \leq j \leq d \\
j \text { odd }}}\left(a_{d-j}-1\right) & =a_{d}-\left\lfloor\frac{d+1}{2}\right\rfloor \\
& =a_{d}-1-\left\lfloor\frac{d}{2}\right\rfloor
\end{aligned}
$$

and if $d$ is even, we also have

$$
\sum_{\substack{1 \leq j \leq d \\ j \text { odd }}}\left(a_{d-j}-1\right)=\left(a_{d}-1\right)-\left\lfloor\frac{d}{2}\right\rfloor .
$$

By duality, $\left(S^{d}\right)^{*}$ is a linearly independent set of linear forms that are non-negative for all $d$-polytopes and 0 for all simple $d$-polytopes, and the intersection of the zero sets of these forms is precisely the span of the flag $f$-vectors of simple polytopes. 
Corollary 1. In the closed convex hull of the flag $f$-vectors of d-polytopes, those of simplicial and simple polytopes span faces of dimension $\lfloor d / 2\rfloor$.

\section{3. $\quad k$-Simplicial and $k$-Simple Polytopes}

Now we can extend this argument to the more general setting of $k$-simplicial polytopes. A polytope is $k$-simplicial if all of its $k$-faces are $k$-simplices, and the affine span of the flag $f$-vectors of these polytopes has dimension $\lfloor d / 2\rfloor+\sum_{i=k+1}^{d-1}\lfloor i / 2\rfloor a_{d-i-1}$, as shown by Kalai in [18]. We would like a linearly independent set $T^{d}$ of linear forms that are non-negative for all $d$-polytopes with zero sets whose intersection is precisely the span of the flag $f$-vectors of $k$-simplicial $d$-polytopes. We work by induction on $d$ for a fixed $k$. As in the simplicial case, we look at dimensions $d-j$ where $j$ is odd. If $d-j \leq k$ we lift inequalities that are equalities for the $(d-j)$-simplex, and if $d-j>k$, we lift inequalities that are equalities for the $k$-simplicial $(d-j)$-polytopes. Our base case is $d=k+1$, so $k$-simplicial is the same as simplicial, and we let $T^{d}=S^{k+1}$ as constructed above.

Suppose we have the desired $T^{m}$ for all $k+1 \leq m \leq d-1$. Let $S_{j}$ be as above for $d-k \leq j \leq d, j$ odd. For $1 \leq j \leq d-k-1, j$ odd, let $T_{j}=\left\{Y^{d-j} y_{j} \mid Y^{d-j} \in T^{d-j}\right\}$. Let

$$
T^{d}=\left(\bigcup_{\substack{d-k \leq j \leq d \\ j \text { odd }}} S_{j}\right) \cup\left(\bigcup_{\substack{1 \leq j \leq d-k-1 \\ j \text { odd }}} T_{j}\right)
$$

Theorem 4. $T^{d}$ is a linearly independent set of linear forms that are non-negative for all d-polytopes and vanish on $k$-simplicial d-polytopes. The intersection of the zero sets of these forms is precisely the span of the flag $f$-vectors of $k$-simplicial polytopes.

Proof. By the same arguments used in the simplicial case, we have non-negativity and linear independence. Also as above, we know that the elements of the $S_{j}$ 's are 0 for $k$-simplicial $d$-polytopes. Consider $Y^{d-j} y_{j} \in T_{j}, 1 \leq j \leq d-k-1$. $Y^{d-j}$ is 0 on $k$-simplicial $(d-j)$-polytopes. Every $(d-j)$-face of a $k$-simplicial $d$-polytope is $k$-simplicial and hence $Y^{d-j} y_{j}$ is 0 for all $k$-simplicial $d$-polytopes.

Now we show that $T^{d}$ has the correct size. We have

$$
\begin{aligned}
\left|T^{d}\right|= & \sum_{\substack{d-k \leq j \leq d \\
j \text { odd }}}\left|S_{j}\right|+\sum_{\substack{1 \leq j \leq d-k-1 \\
j \text { odd }}}\left|T_{j}\right| \\
= & \sum_{\substack{d-k \leq j \leq d \\
j \text { odd }}}\left(a_{d-j}-1\right) \\
& +\sum_{\substack{1 \leq j \leq d-k-1 \\
j \text { odd }}}\left(\left(a_{d-j}-1\right)-\left(\left\lfloor\frac{(d-j)}{2}\right\rfloor+\sum_{i=k+1}^{d-j-1}\left\lfloor\frac{i}{2}\right\rfloor a_{d-j-i-1}\right)\right)
\end{aligned}
$$




$$
\begin{aligned}
& =\sum_{\substack{1 \leq j \leq d \\
j \text { odd }}}\left(a_{d-j}-1\right)-\sum_{\substack{1 \leq j \leq d-k-1 \\
j \text { odd }}}\left(\left\lfloor\frac{(d-j)}{2}\right\rfloor+\sum_{i=k+1}^{d-j-1}\left\lfloor\frac{i}{2}\right\rfloor a_{d-j-i-1}\right) \\
& =a_{d}-1-\left\lfloor\frac{d}{2}\right\rfloor-\sum_{\substack{k+1 \leq i \leq d-1 \\
d-i \text { odd }}}\left\lfloor\frac{i}{2}\right\rfloor-\sum_{\substack{1 \leq j \leq d-k-1 \\
j \text { odd }}} \sum_{\substack{i=k+1 \\
\text { od }}}^{d-j-1}\left\lfloor\frac{i}{2}\right\rfloor a_{d-j-i-1} \\
& =a_{d}-1-\left\lfloor\frac{d}{2}\right\rfloor-\sum_{\substack{k+1 \leq i \leq d-1 \\
d-i \text { odd }}}\left\lfloor\frac{i}{2}\right\rfloor-\sum_{i=k+1}^{d-2}\left\lfloor\frac{i}{2}\right\rfloor\left(\sum_{\substack{1 \leq j \leq d-i-1 \\
j \text { odd }}} a_{d-i-j-1}\right) \\
& =a_{d}-1-\left\lfloor\frac{d}{2}\right\rfloor-\sum_{i=k+1}^{d-1}\left\lfloor\frac{i}{2}\right\rfloor a_{d-i-1},
\end{aligned}
$$

as desired. Equation (5) follows from (4) by the same Fibonacci identity we used in the simplicial case and by letting $i=d-j$ in the second sum. We use the Fibonacci identity again to get (7) from (6). Recall that the identity requires the extra 1 when $d-i-j$ is even; since $j$ is always odd, this happens when $d-i$ is also odd, which is precisely when the first sum contributes $\lfloor i / 2\rfloor$.

Dual to $k$-simplicial polytopes are $k$-simple polytopes, whose vertex figures are $k$ simplicial. By duality, $\left(T^{d}\right)^{*}$ is a linearly independent set of linear forms that are nonnegative for all $d$-polytopes and 0 for all $k$-simple $d$-polytopes, and the intersection of the zero sets of these forms is precisely the span of the flag $f$-vectors of $k$-simple polytopes.

Corollary 2. In the closed convex hull of the flag $f$-vectors of $d$-polytopes, those of $k$ simplicial and $k$-simple polytopes span faces of dimension $\lfloor d / 2\rfloor+\sum_{i=k+1}^{d-1}\lfloor i / 2\rfloor a_{d-i-1}$.

We should note that it is also possible to extend Kalai's work in [18] to find facedefining inequalities for the flag $f$-vectors of $k$-simplicial polytopes. To do this, use Kalai's Lemma 5.1, the toric $g$-system described in his Section 6, and Karu's recent proof [19] that the toric $g$-vector is non-negative for all polytopes. Our approach is somewhat longer, but it is valuable in its own right because of the connections it draws between the convex hulls of flag $f$-vectors of polytopes of various dimensions and because of its use of the Billera-Liu algebra. It also extends quite naturally to the cone of flag $f$-vectors of zonotopes, as we show in the next section. It would be interesting to see what other families of polytopes fit nicely into this framework.

\section{Cubical and $k$-Cubical Zonotopes}

We can use the same techniques to study cubical zonotopes, which, not surprisingly, are zonotopes whose facets are all cubes. However, first we must show 
Theorem 5. The span of the flag $f$-vectors of cubical $d$-zonotopes has dimension $\lfloor d / 2\rfloor$.

Proof. We know $\lfloor d / 2\rfloor$ is an upper bound on the dimension, since it is the dimension of the span of the $f$-vectors of all cubical $d$-polytopes [17, Section 9.4]. As in the simplicial case, the flag $f$-vector of a cubical polytope is completely determined by the $f$-vector.

The flag $f$-vector of a cubical zonotope depends only on the number of zones it has. (See [26] for a discussion.) Buck [14] and Zaslavsky [26] showed that if $Z$ is a cubical $d$-zonotope with $n$ zones then

$$
f_{i}=2 \sum_{j=0}^{\lfloor(d-1-i) / 2\rfloor}\left(\begin{array}{c}
n \\
d-1-2 j
\end{array}\right)\left(\begin{array}{c}
d-1-2 j \\
i
\end{array}\right) .
$$

To show that the dimension of the span of the flag $f$-vectors is at least (and therefore exactly) $\lfloor d / 2\rfloor$, we take $\lfloor d / 2\rfloor$ of these $f_{i}$ and show that they cannot satisfy any linear relation.

If $d$ is even, let $U=\left\{f_{d-1}, f_{d-3}, \ldots, f_{1}\right\}$, and if $d$ is odd, let $U=\left\{f_{d-1}, f_{d-3}, \ldots\right.$, $\left.f_{2}\right\}$. In either case, $|U|=\lfloor d / 2\rfloor$. We can think of $\left(\begin{array}{l}n \\ k\end{array}\right)$ as a degree $k$ polynomial in $n$. Then $\left\{\left(\begin{array}{l}n \\ 0\end{array}\right),\left(\begin{array}{l}n \\ 1\end{array}\right), \ldots,\left(\begin{array}{l}n \\ k\end{array}\right), \ldots\right\}$ is a basis for the vector space over $\mathbf{R}$ of polynomials in $n$. Equation (8) expresses $f_{i}$ as a linear combination of the basis elements $\left(\begin{array}{c}n \\ d-1\end{array}\right), \ldots,\left(\begin{array}{c}n \\ i\end{array}\right)$, and the coefficient of $\left(\begin{array}{l}n \\ i\end{array}\right)$ is 1 . So the elements of $U$ are linearly independent, and therefore the span of the flag $f$-vectors of cubical zonotopes has dimension $\lfloor d / 2\rfloor$.

Now we proceed as in the simplicial case. Let $Y^{d-j}$ be a linear form written in terms of the $y_{2 k+1}$ 's that is non-negative for all $(d-j)$-zonotopes and 0 for the $(d-j)$ cube. For each odd $j$ in $\{1, \ldots, d\}$, pick a set $X_{j}$ of $a_{d-j}-1$ linearly independent such $Y^{d-j}$. We know that these $Y^{d-j}$ exist because the flag $f$-vector of the cube is a vertex of the cone of flag $f$-vectors of zonotopes [7]. Let $Z_{j}=\left\{Y^{d-j} y_{j} \mid Y^{d-j} \in X_{j}\right\}$. Let $Z^{d}=\bigcup_{1 \leq j \leq d, j \text { odd }} Z_{j}$. Then, with minor modification, the proof of Theorem 3 gives us

Theorem 6. $\quad Z^{d}$ is a linearly independent set of linear forms that are non-negative for all $d$-zonotopes and vanish on cubical d-polytopes. The intersection of the zero sets of these forms is precisely the span of the flag $f$-vectors of cubical zonotopes.

Corollary 3. In the closed convex hull of the flag $f$-vectors of $d$-zonotopes, those of cubical zonotopes span a face of dimension $\lfloor d / 2\rfloor$.

There are also simple zonotopes; the $d$-cube, for example, is simple. The dimension of the span of the flag $f$-vectors of simple zonotopes is not known. It is bounded above by $\lfloor d / 2\rfloor$, the dimension of the span of the flag $f$-vectors of all simple polytopes.

We call a zonotope $k$-cubical if all of its $k$-dimensional faces are cubes. Just as cubical zonotopes are analogous to simplicial polytopes, $k$-cubical zonotopes are analogous to $k$-simplicial polytopes. The dimension of the span of the flag $f$-vectors of $k$-cubical zonotopes is not known. However, we can use the techniques of Section 3 to get an upper bound on this dimension. 
We want a linearly independent set $W^{d}$ of linear forms that are non-negative for all $d$-zonotopes with zero sets whose intersection contains the flag $f$-vectors of $k$-cubical $d$-zonotopes. Again we work by induction on $d$ for a fixed $k$, and again we look at dimensions $d-j$ where $j$ is odd. If $d-j \leq k$ we lift inequalities that are equalities for the $(d-j)$-cube, and if $d-j>k$, we lift inequalities that are equalities for the $k$-cubical $(d-j)$-zonotopes. Our base case is $d=k+1$, so $k$-cubical is the same as cubical, and we let $W^{d}=Z^{k+1}$ as constructed above.

Suppose we have the desired $W^{m}$ for all $k+1 \leq m \leq d-1$. For $1 \leq j \leq d-k-1$, $j$ odd, let $W_{j}=\left\{Y^{d-j} y_{j} \mid Y^{d-j} \in W^{d-j}\right\}$. Let

$$
W^{d}=\left(\bigcup_{\substack{d-k \leq j \leq d \\ j \text { odd }}} Z_{j}\right) \cup\left(\bigcup_{\substack{1 \leq j \leq d-k-1 \\ j \text { odd }}} W_{j}\right) .
$$

Then by the same arguments used in the proof of Theorem 4, we have

Theorem 7. $\quad W^{d}$ is a linearly independent set of linear forms that are non-negative for all $d$-zonotopes and vanish on $k$-cubical d-zonotopes. The intersection of the zero sets of these forms contains the flag $f$-vectors of $k$-cubical $d$-zonotopes. The dimension of this intersection is $\lfloor d / 2\rfloor+\sum_{i=k+1}^{d-1}\lfloor i / 2\rfloor a_{d-i-1}$, which is an upper bound on the dimension of the span of the flag $f$-vectors of $k$-cubical $d$-zonotopes.

If the parallel with $k$-simplicial polytopes continues, we expect this upper bound to be the actual dimension of the span of the flag $f$-vectors of $k$-cubical $d$-zonotopes. To prove this, we would need a basis of $k$-cubical zonotopes. Possible approaches to this problem include modifying the work of Kalai in [18] or the work of Billera et al. in [8].

\section{More on Zonotopes}

We have seen that in some ways the flag $f$-vectors of zonotopes behave much like the flag $f$-vectors of all polytopes. For example, the flag $f$-vectors of cubical zonotopes fit into the convex hull of the flag $f$-vectors of all zonotopes just as those of simplicial polytopes fit into the convex hull of the flag $f$-vectors of all polytopes. However, Varchenko [25] and Liu [20] showed that the flag $f$-vectors of zonotopes also satisfy some inequalities not satisfied by all polytopes, and these inequalities do not seem to have an obvious generalization that would hold for all polytopes. We strengthen their results.

Varchenko and Liu's inequalities derive from the fact that zonotopes are dual to hyperplane arrangements. A hyperplane arrangement $H$ is a collection of hyperplanes, or $(d-1)$-dimensional subspaces, in $d$-space. If each hyperplane contains the origin, the arrangement is central; all arrangements discussed here are central. Each hyperplane has a normal vector, and the Minkowski sum of these normal vectors is the dual zonotope $Z$. For further discussion of this duality, see [26] or [27]. A central hyperplane arrangement breaks $d$-space into $d$-dimensional polyhedra, so it has a flag $f$-vector. A $k$-face of $Z$ corresponds to a $(d-k)$-face of $H$. 
Varchenko showed the following:

Theorem 8 [25]. Let $H$ be a central hyperplane arrangement in $\mathbf{R}^{\mathbf{d}}$. Let $f_{k}$ be the number of $k$-dimensional faces of the arrangement, and let $f_{j, k}$ be the number of $\{j, k\}$ flags in the arrangement. For $1 \leq j<k \leq d$, we have

$$
f_{j, k}(H) \leq\left(\begin{array}{c}
k-1 \\
j-1
\end{array}\right) 2^{k-j} f_{k}(H)
$$

Liu extended Varchenko's work by considering all possible flags of faces in the arrangement.

Theorem 9 [20]. Let $H$ be a central hyperplane arrangement in $\mathbf{R}^{\mathbf{d}}$. Let $S=\left\{i_{1}\right.$, $\left.i_{2}, \ldots, i_{k}\right\}$ be a subset of $\{1,2, \ldots, d\}$ with $k \geq 2$. Then

$$
f_{S}(H) \leq\left(\begin{array}{c}
i_{k} \\
i_{1}, i_{2}-i_{1}, \ldots, i_{k}-i_{k-1}
\end{array}\right) 2^{i_{k}-i_{1}} f_{i_{k}}(H)
$$

By duality, this means

Corollary 4 [20]. Let $Z$ be a d-zonotope. Let $S=\left\{i_{1}, i_{2}, \ldots, i_{k}\right\}$ be a subset of $\{0,1, \ldots, d-1\}$ with $k \geq 2$. Then

$$
f_{S}(Z) \leq\left(\begin{array}{c}
d-i_{1} \\
i_{2}-i_{1}, \ldots, i_{k}-i_{k-1}, d-i_{k}
\end{array}\right) 2^{i_{k}-i_{1}} f_{i_{1}}(Z)
$$

We modify Liu's proof to tighten his result.

Theorem 10. Let $H$ be a central hyperplane arrangement in $\mathbf{R}^{\mathbf{d}}$ and let $S=\left\{i_{1}\right.$, $\left.i_{2}, \ldots, i_{k}\right\}$ be a subset of $\{1,2, \ldots, d\}$ with $k \geq 2$. Then

$$
f_{S}(H) \leq\left(\begin{array}{c}
i_{k}-1 \\
i_{1}-1, i_{2}-i_{1}, \ldots, i_{k}-i_{k-1}
\end{array}\right)\left(2^{i_{k}-i_{1}} f_{i_{k}}(H)-2^{i_{k}}\left(\begin{array}{l}
d \\
i_{k}
\end{array}\right)\left(2^{i_{k}-i_{1}}-i_{k} / i_{1}\right)\right) .
$$

To prove this, we need more information about hyperplane arrangements. If $H=$ $\left\{H_{1}, \ldots, H_{n}\right\}$ is a hyperplane arrangement in $\mathbf{R}^{d}$ and $A$ is a hyperplane not in $H$, we define $H \cap A=\left\{H_{1} \cap A, \ldots, H_{n} \cap A\right\}$, a hyperplane arrangement in $\mathbf{R}^{d-1}$. We also define $H \cup A=\left\{H_{1}, \ldots, H_{n}, A\right\}$. Consider a $d$-face $F$ of $H$ such that $A$ intersects the interior of $F$. Then $A$ cuts $F$ into two $d$-faces of $H \cup A$, and the intersection of these two $d$-faces is a $(d-1)$-face of $H \cap A$. Conversely, each $(d-1)$-face of $H \cap A$ comes from the intersection of $A$ with the interior of a single $d$-face of $H$. Thus we have

$$
f_{d}(H \cup A)=f_{d}(H)+f_{d-1}(H \cap A) .
$$


We also need the following lemma:

Lemma 1 [20]. Let $H$ be a central hyperplane arrangement in $\mathbf{R}^{\mathbf{d}}$ and let $A$ be a hyperplane not in $H$. Let $S=\left\{i_{1}, i_{2}, \ldots, i_{k}\right\}$ be a subset of $\{1,2, \ldots, d\}$ with $i_{k}=d$ and $k \geq 2$. Then

$$
\begin{aligned}
f_{S}(H \cup A) \leq & f_{S}(H)+\sum_{j=2}^{k} 2 f_{i_{1}, \ldots, i_{j-1}, i_{j}-1, \ldots, i_{k}-1}(H \cap A) \\
& +f_{i_{1}-1, i_{2}-1, \ldots, i_{k}-1}(H \cap A) .
\end{aligned}
$$

Proof of Theorem 10. Case 1. First, we prove the case $i_{k}=d$ by double induction on $d$ and the number of hyperplanes. Note $\left(\begin{array}{l}d \\ d\end{array}\right)=1$, so we leave this term out to simplify the calculations. For the base case let $H^{d}$ be $d$ hyperplanes in general position in $\mathbf{R}^{\mathbf{d}}$, for example the coordinate hyperplanes. Then $f_{d}\left(H^{d}\right)=2^{d}$ and

$$
f_{i_{1}, i_{2}, \ldots, i_{k-1}, d}\left(H^{d}\right)=\left(\begin{array}{c}
d \\
i_{1}, i_{2}-i_{1}, \ldots, i_{k-1}-i_{k-2}, d-i_{k-1}
\end{array}\right) 2^{d} .
$$

So we have

$$
f_{i_{1}, i_{2}, \ldots, i_{k-1}, d}\left(H^{d}\right)=\left(\begin{array}{c}
d-1 \\
i_{1}-1, i_{2}-i_{1}, \ldots, d-i_{k-1}
\end{array}\right)\left(2^{d-i_{1}} 2^{d}-2^{d}\left(2^{d-i_{1}}-d / i_{1}\right)\right)
$$

and the theorem is true (with equality) for $H^{d}$.

If $H$ has more than $d$ hyperplanes, choose any $A \in H$ and let $G=H \backslash A$. By Lemma 1 , we have

$$
\begin{aligned}
f_{S}(H) & =f_{S}(G \cup A) \\
& \leq f_{S}(G)+\sum_{j=2}^{k} 2 f_{i_{1}, \ldots, i_{j-1}, i_{j}-1, \ldots, i_{k}-1}(G \cap A)+f_{i_{1}-1, i_{2}-1, \ldots, i_{k}-1}(G \cap A) .
\end{aligned}
$$

Now we apply the induction hypothesis to $f_{S}(G)$ and a weaker form of the hypothesis (omitting the subtracted constant to simplify the calculation) to each $f_{T}(G \cap A)$.

$$
\begin{aligned}
f_{S}(H) \leq & \left(\begin{array}{c}
i_{k}-1 \\
i_{1}-1, i_{2}-i_{1}, \ldots, i_{k}-i_{k-1}
\end{array}\right)\left(2^{i_{k}-i_{1}} f_{i_{k}}(G)-2^{i_{k}}\left(2^{i_{k}-i_{1}}-i_{k} / i_{1}\right)\right) \\
& +2 \sum_{j=2}^{k}\left(\begin{array}{c}
i_{k}-2 \\
i_{1}-1, \ldots, i_{j-1}-i_{j-2}, i_{j}-i_{j-1}-1, i_{j+1}-i_{j}, \ldots, i_{k}-i_{k-1}
\end{array}\right) \\
& \cdot 2^{\left(i_{k}-1\right)-i_{1}} f_{i_{k}-1}(G \cap A) \\
& +\left(\begin{array}{c}
i_{k}-2 \\
i_{1}-2, i_{2}-i_{1}, \ldots, i_{k}-i_{k-1}
\end{array}\right) 2^{\left(i_{k}-1\right)-\left(i_{1}-1\right)} f_{i_{k}-1}(G \cap A) .
\end{aligned}
$$

We use the multinomial identity

$$
\left(\begin{array}{c}
n \\
n_{1}, n_{2}, \ldots, n_{k}
\end{array}\right)=\sum_{j=1}^{k}\left(\begin{array}{c}
n-1 \\
n_{1}, \ldots, n_{j-1}, n_{j}-1, n_{j+1}, \ldots, n_{k}
\end{array}\right)
$$


to obtain

$$
\begin{aligned}
f_{S}(H) \leq & \left(\begin{array}{c}
i_{k}-1 \\
i_{1}-1, i_{2}-i_{1}, \ldots, i_{k}-i_{k-1}
\end{array}\right) \\
& \cdot\left(2^{i_{k}-i_{1}}\left(f_{i_{k}}(G)+f_{i_{k-1}}(G \cap A)\right)-2^{i_{k}}\left(2^{i_{k}-i_{1}}-i_{k} / i_{1}\right)\right) .
\end{aligned}
$$

Applying (13) to $H=G \cup A$, we have

$$
f_{S}(H) \leq\left(\begin{array}{c}
i_{k}-1 \\
i_{1}-1, i_{2}-i_{1}, \ldots, i_{k}-i_{k-1}
\end{array}\right)\left(2^{i_{k}-i_{1}} f_{i_{k}}(H)-2^{i_{k}}\left(2^{i_{k}-i_{1}}-i_{k} / i_{1}\right)\right)
$$

so the theorem holds for $i_{k}=d$.

Case 2. When $i_{k}<d$, let $\mathcal{F}=\{F \mid F$ is an intersection of some hyperplanes in $\left.H, \operatorname{dim}(F)=i_{k}\right\}$. For each $F \in \mathcal{F}, H \cap F$ is an $i_{k}$-dimensional hyperplane arrangement. Hence, by the previous case

$$
\begin{aligned}
& f_{i_{1}, i_{2}, \ldots, i_{k-1}, i_{k}}(H \cap F) \\
& \quad \leq\left(\begin{array}{c}
i_{k}-1 \\
i_{1}-1, i_{2}-i_{1}, \ldots, i_{k}-i_{k-1}
\end{array}\right)\left(2^{i_{k}-i_{1}} f_{i_{k}}(H \cap F)-2^{i_{k}}\left(2^{i_{k}-i_{1}}-i_{k} / i_{1}\right)\right) .
\end{aligned}
$$

Note that $f_{S}(H)=\sum_{F \in \mathcal{F}^{i_{k}}} f_{S}(H \cap F)$ and $f_{i_{k}}(H)=\sum_{F \in \mathcal{F}^{i k}} f_{i_{k}}(H \cap F)$. So

$$
\begin{aligned}
& f_{i_{1}, i_{2}, \ldots, i_{k-1}, i_{k}}(H) \\
& \quad \leq\left(\begin{array}{c}
i_{k}-1 \\
i_{1}-1, i_{2}-i_{1}, \ldots, i_{k}-i_{k-1}
\end{array}\right)\left(2^{i_{k}-i_{1}} f_{i_{k}}(H)-|\mathcal{F}| 2^{i_{k}}\left(2^{i_{k}-i_{1}}-i_{k} / i_{1}\right)\right) .
\end{aligned}
$$

Since $H$ contains at least $d$ hyperplanes,

$$
|\mathcal{F}| \geq\left(\begin{array}{c}
d \\
d-i_{k}
\end{array}\right)=\left(\begin{array}{l}
d \\
i_{k}
\end{array}\right)
$$

and the theorem holds.

Since zonotopes are dual to hyperplane arrangements, we have the following:

Corollary 5. If $Z$ is a $d$-zonotope and $S=\left\{i_{1}, i_{2}, \ldots, i_{k}\right\} \subset\{0,1, \ldots, d-1\}$ then the following is an upper bound for $f_{S}(Z)$ :

$$
\left(\begin{array}{c}
d-i_{1}-1 \\
i_{2}-i_{1}, \ldots, i_{k}-i_{k-1}, d-i_{k}-1
\end{array}\right)\left(2^{i_{k}-i_{1}} f_{i_{1}}(Z)-2^{d-i_{1}}\left(\begin{array}{l}
d \\
i_{1}
\end{array}\right)\left(2^{i_{k}-i_{1}}-\frac{d-i_{1}}{d-i_{k}}\right)\right) .
$$

Theorem 11. For sufficiently large d, the inequalities of Corollary 5 are not valid for the $d$-simplex $\Delta^{d}$ and hence are not true for polytopes in general.

Proof. Note that

$$
f_{i_{1}}\left(\Delta^{d}\right)=\left(\begin{array}{l}
d+1 \\
i_{1}+1
\end{array}\right)
$$


and

$$
f_{S}\left(\Delta^{d}\right)=\left(\begin{array}{c}
d+1 \\
i_{1}+1, i_{2}-i_{1}, \ldots, i_{k}-i_{k-1}, d-i_{k}
\end{array}\right) .
$$

Fix $i_{1}, \ldots, i_{k}$. Then it is enough to show that for sufficiently large $d$,

$$
\begin{aligned}
& \left(\begin{array}{c}
d+1 \\
i_{1}+1, i_{2}-i_{1}, \ldots, i_{k}-i_{k-1}, d-i_{k}
\end{array}\right) \\
& >\left(\begin{array}{c}
d-i_{1}-1 \\
i_{2}-i_{1}, \ldots, i_{k}-i_{k-1}, d-i_{k}-1
\end{array}\right) \\
& \times\left(2^{i_{k}-i_{1}}\left(\begin{array}{l}
d+1 \\
i_{1}+1
\end{array}\right)-2^{d-i_{1}}\left(\begin{array}{l}
d \\
i_{1}
\end{array}\right)\left(2^{i_{k}-i_{1}}-\frac{d-i_{1}}{d-i_{k}}\right)\right) .
\end{aligned}
$$

Write the two multinomial coefficients out in terms of factorials and cancel the $\left(i_{2}-i_{1}\right)$ ! through $\left(i_{k}-i_{k-1}\right)$ ! terms to get

$$
\begin{aligned}
& \frac{(d+1) !}{\left(i_{1}+1\right) !\left(d-i_{k}\right) !} \\
& \quad>\frac{\left(d-i_{1}-1\right) !}{\left(d-i_{k}-1\right) !}\left(2^{i_{k}-i_{1}}\left(\begin{array}{l}
d+1 \\
i_{1}+1
\end{array}\right)-2^{d-i_{1}}\left(\begin{array}{l}
d \\
i_{1}
\end{array}\right)\left(2^{i_{k}-i_{1}}-\frac{d-i_{1}}{d-i_{k}}\right)\right) .
\end{aligned}
$$

This is equivalent to

$$
\begin{aligned}
\frac{(d+1)(d) \cdots\left(d-i_{1}\right)}{\left(i_{1}+1\right) !\left(d-i_{k}\right)} & =\left(\begin{array}{l}
d+1 \\
i_{1}+1
\end{array}\right)\left(\frac{d-i_{1}}{d-i_{k}}\right) \\
& >2^{i_{k}-i_{1}}\left(\begin{array}{l}
d+1 \\
i_{1}+1
\end{array}\right)-2^{d-i_{1}}\left(\begin{array}{l}
d \\
i_{1}
\end{array}\right)\left(2^{i_{k}-i_{1}}-\frac{d-i_{1}}{d-i_{k}}\right)
\end{aligned}
$$

which is equivalent to

$$
2^{d-i_{1}}\left(\begin{array}{l}
d \\
i_{1}
\end{array}\right)\left(2^{i_{k}-i_{1}}-\frac{d-i_{1}}{d-i_{k}}\right)>\left(\begin{array}{l}
d+1 \\
i_{1}+1
\end{array}\right)\left(2^{i_{k}-i_{1}}-\frac{d-i_{1}}{d-i_{k}}\right)
$$

which is true provided

$$
2^{i_{k}-i_{1}}-\frac{\left(d-i_{1}\right)}{\left(d-i_{k}\right)}>0
$$

and

$$
2^{d-i_{1}}\left(\begin{array}{l}
d \\
i_{1}
\end{array}\right)>\left(\begin{array}{l}
d+1 \\
i_{1}+1
\end{array}\right)
$$

and both of these inequalities hold for sufficiently large $d$.

\section{Further Questions}

We have found inequalities that define the simplicial, simple, $k$-simplicial, and $k$-simple faces of the convex hull of flag $f$-vectors of $d$-polytopes. Our proof involved lifting 
vertex-defining inequalities from lower dimensions by convolving them with the appropriate $f_{\emptyset}^{j}$. How else can we use lower-dimensional inequalities to understand the faces of the convex hull of the flag $f$-vectors?

For example, Billera and Hetyei [9] studied $K_{n}$, the cone of all linear forms that are non-negative for all graded posets of rank $n$. They showed that if $F$ and $G$ are extreme rays of $K_{m}$ and $K_{n}$, respectively, then $F * G$ is an extreme ray of $K_{m+n}$ unless $F=F^{\prime} * f_{\emptyset}^{k}$ and $G=f_{\emptyset}^{l} * G^{\prime}$ for some $k \leq m, l \leq n, F^{\prime} \in K_{m-k}$, and $G^{\prime} \in K_{n-l}$. Note that if $F$ is an extreme ray of $K_{m}$, then $F \geq 0$ defines a facet of the closure of the convex hull of flag $f$-vectors of graded posets of rank $m$. So the convolution of two facet-defining inequalties yields a facet-defining inequality except in the case described above. Is there a similar statement for flag $f$-vectors of polytopes?

\section{Acknowledgments}

Many thanks to Lou Billera for his suggestions and comments on this work. Thanks also to two anonymous referees for their insights.

\section{References}

1. R. Adin, A new cubical $h$-vector, Discrete Math. 157 (1996), 3-14.

2. M. Aguiar, Infinitesimal Hopf algebras and the cd-index of polytopes, Discrete Comput. Geom. 27(1) (2002), 3-28.

3. M. Bayer and L. J. Billera, Generalized Dehn-Sommerville relations for polytopes, spheres and Eulerian partially ordered sets, Invent. Math. 79 (1985), 143-157.

4. M. Bayer and A. Klapper, A new index for polytopes, Discrete Comput. Geom. 6 (1991), 33-47.

5. N. Bergeron, S. Mykytiuk, F. Sottile, and S. van Willigenburg, Non-commutative Pieri operators on posets, J. Combin. Theory Ser. A 91 (2000), 84-110.

6. L. J. Billera and R. Ehrenborg, Monotonicity of the cd-index for polytopes, Math. Z. 233 (2000), 421-441.

7. L. J. Billera, R. Ehrenborg, and M. Readdy, The c-2d-index of oriented matroids, J. Combin. Theory Ser. A 80 (1997), 79-105.

8. L. J. Billera, R. Ehrenborg, and M. Readdy, The cd-index of zonotopes and arrangements, in Mathematical Essays in Honor of Gian-Carlo Rota, pp. 23-40, Birkhäuser, Boston, MA, 1998.

9. L. J. Billera and G. Hetyei, Linear inequalities for flags in graded partially ordered sets, J. Combin. Theory Ser. A 89 (2000), 77-104.

10. L. J. Billera, S. K. Hsiao, and S. van Willigenburg, Peak quasisymmetric functions and Eulerian enumeration, Adv. Math. 176 (2003), 248-276.

11. L. J. Billera and C. Lee, Sufficiency of McMullen's conditions for $f$-vectors of simplicial polytopes, Bull. Amer. Math. Soc. 2 (1980), 181-185.

12. L. J. Billera and C. Lee, A proof of the sufficiency of McMullen's conditions for $f$-vectors of simplicial polytopes, J. Combin. Theory Ser. A 31 (1981), 237-255.

13. L. J. Billera and N. Liu, Noncommutative enumeration in graded posets, J. Algebraic Combin. 12 (2000), $7-24$.

14. R. C. Buck, Partition of space, Amer. Math. Monthly 50 (1943), 541-544.

15. R. Ehrenborg, On posets and Hopf algebras, Adv. Math. 119 (1996), 1-25.

16. R. Ehrenborg, Lifting inequalities for polytopes, Adv. Math. 193 (2005), 205-222.

17. B. Grünbaum, Convex Polytopes, Wiley, London, 1967.

18. G. Kalai, A new basis of polytopes, J. Combin. Theory Ser. A 49 (1988), 191-208.

19. K. Karu, Hard Lefschetz theorem for nonrational polytopes, Invent. Math. 157(2) (2004), 419-447.

20. N. Liu, Algebraic and Combinatorial Methods for Face Enumeration in Polytopes, Ph.D. Thesis, Cornell University, Ithaca, NY, May 1995. 
21. R. Stanley, The number of faces of a simplicial convex polytope, Adv. Math. 35 (1980), 236-238.

22. R. Stanley, Generalized $h$-vectors, intersection cohomology of toric varieties, and related results, in Commutatitve Algebra and Combinatorics, pp. 187-213, Advanced Studies in Pure Mathematics, vol. 11, Kinokunia, Tokyo, and North-Holland, Amsterdam, 1987.

23. R. Stanley, Flag $f$-vectors and the cd-index, Math. Z. 216 (1994), 483-499.

24. E. Steinitz, Über die Eulersche Polyederrelationen, Arch. Math. Phys. (3) 11 (1906), 86-88.

25. A. N. Varchenko, On the numbers of faces of a configuration of hyperplanes, Soviet Math. Dokl. 38(2) (1989), 291-295.

26. T. Zaslavsky, Facing up to arrangements: face-count formulas for partitions of space by hyperplanes, Mem. Amer. Math. Soc. 1, No. 154 (1975).

27. G. Ziegler, Lectures on Polytopes, Graduate Texts in Mathematics, Vol. 152, Springer-Verlag, New York, 1995.

Received October 6, 2003, and in revised form June 30, 2004, and May 27, 2005.

Online publication August 2, 2005. 